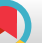

\title{
An Investigation of the Factor Structure of the Persian Version of the Dysphagia Handicap Index
}

\author{
Ebrahim Barzegar Bafrooei ${ }^{1}$, Seyyed Ahmadreza Khatoonabadi ${ }^{2}$, Saman Maroufizadeh ${ }^{3}$, Jalal \\ Bakhtiyari (iD ${ }^{4,}$ and Ali rajaee ${ }^{5}$ \\ ${ }^{1}$ Department of Speech Therapy, University of Social Welfare and Rehabilitation Sciences, Tehran, Iran \\ ${ }^{2}$ Department of Speech Therapy, School of Rehabilitation, Tehran University of Medical Sciences, Tehran, Iran \\ ${ }^{3}$ Department of Biostatistics, School of Nursing and Midwifery, Guilan University of Medical Sciences, Rasht, Iran \\ ${ }^{4}$ Neuromuscular Rehabilitation Research Center, Semnan University of Medical Sciences, Semnan, Iran \\ ${ }^{5}$ Isfahan Neurosciences Research Center, Al-Zahra Hospital Isfahan University of Medical Sciences, Isfahan, Iran \\ "Corresponding author: Neuromuscular Rehabilitation Research Center, Semnan University of Medical Sciences, Semnan, Iran. Email: bakhtiyari.slp@gmail.com
}

Received 2020 May 05; Revised 2020 June 20; Accepted 2020 June 22.

\begin{abstract}
Background: Oropharyngeal dysphagia harms all aspects of a person's life, including behaviors, activities, and social situations. Quality of life is a multidimensional concept. The dysphagia handicap index (DHI) is a patient-reported outcomes tool that evaluates the different dimensions of quality of life in three physical, emotional, and functional factors in English-speaking countries. The validity of the Persian version of the dysphagia handicap index (DHI) has been evaluated in a study, but the reliability and factor analysis of the Persian version has not been investigated in any study.

Objectives: This study aimed to investigate the factor structure analysis and reliability of the Persian version of the dysphagia handicap index (P-DHI).

Methods: In this cross-sectional study, 100 patients with oropharyngeal dysphagia (mean age $55.69 \pm 15.04,53$ women) completed the dysphagia handicap index (DHI). The severity of dysphagia was defined as follows: 1 for no dysphagia (normal), 2 and 3 for mild, 4 , and 5 for moderate and 6 and 7for severe. Construct validity was examined by confirmatory factor analysis (CFA). Cronbach's alpha and intraclass correlation coefficient (ICC) were performed to evaluate the internal consistency and test-retest reliability of the P-DHI, respectively.

Results: The results of CFA provided support for a second-order three-factor model of P-DHI $\left(\chi^{2}=484.61, \mathrm{df}=273,(\mathrm{P}=0.0001), \chi^{2} / \mathrm{df}\right.$ $=1.77 ; \mathrm{CFI}=0.901 ; \mathrm{RMSEA}=0.088$ and SRMR $=0.010)$. The Cronbach's alpha for physical, functional, and emotional subscales and total score were $0.751,0.836,0.773$, and 0.900 , respectively. The test-retest reliability of the P-DHI for the total score and subscales was high (ICC: 0.952 - 0.988). Furthermore, a significant relationship was found between the P-DHI total score and self-reported severity of dysphagia $(\mathrm{P}=0.0001)$, while there were no relationships between $\mathrm{P}-\mathrm{DHI}$ total score and age $(\mathrm{P}=0.223)$, sex $(\mathrm{P}=0.936)$, level of education $(\mathrm{P}=0.113)$, disease duration $(\mathrm{P}=0.126)$.

Conclusions: The P-DHI is a reliable and valid instrument for assessing the disabling effects of swallowing disorders on the one's Quality of Life in Iranian patients with oropharyngeal dysphagia due to a variety of diseases. Also, the CFA findings provide support for the tree-factor structure of the P-DHI and the use of the subscales as distinct variables.
\end{abstract}

Keywords: Deglutition Disorders, Dysphagia, Quality of Life, Persian

\section{Background}

Dysphagia is one of the most common morbidities after a wide range of diseases such as stroke, Parkinson's disease, multiple sclerosis, amyotrophic lateral sclerosis, or traumatic brain injury (1). Swallowing disorder is also a common problem in the elderly due to physiological changes and age-related diseases in the elderly (2). Oropharyngeal dysphagia can lead to aspiration, malnutrition, dehydration, and negative impacts on the quality of life (QOL) (3-5). Patients with dysphagia have reported social and psychological consequences that affect their daily and personal lives, including feelings of anxiety, depression, reduced self-esteem, and social isolation (6).

Although traditional clinician-driven assessments such as videofluoroscopy and fiber optic endoscopic evaluation of swallowing are the gold standards for assessment of swallowing function, they do not consider the patients' view about the problem (7). Thus, Multidimensional dysphagia assessment, including patient-based and objective instruments has been recommended for 
evaluating dysphagia (8). In recent years, researchers have attempted to create new tools such as self-report questionnaires to do a comprehensive self-assessment of dysphagia (9).

Several validated questionnaires have been developed to assess the quality of life in patients with dysphagia to date: the M.D.Anderson Dysphagia inventory(10), the Swallowing Quality of Life questionnaire (11), the eating assessment tool (EAT-10) (12) and the deglutition handicap index (13).

Silbergleit et al. (9), in 2012, created and validated the dysphagia handicap index (DHI). dysphagia handicap index (DHI) has several advantages over other quality of life assessment tools in dysphagia patients, including Easy completion by most patient populations, use clear sentences that indicate patients' complaints and easy daily use to evaluate the effect of dysphagia on three physical, emotional and functional factors of quality of life of dysphagia patient. It is a relatively new self-administered questionnaire that includes 25 items (9). The DHI has been translated into several languages such as Arabic (14), Persian (15), Japanese (16), and Hebrew (17). Factor structure analysis is a statistical method for investigating variable relationships for complex concepts such as socioeconomic status, quality of life, or psychological scales. Quality of life is a multidimensional concept that is measured by the original three factors structure including physical, emotional, and functional of the instruments (3). The original version of the DHI identified three main factors as indicators for determining the effect of dysphagia on patients' quality of life. The validity of the Persian version of the Dysphagia Handicap Index (DHI) has been evaluated in a study (15) but the factor analysis of the Persian version has not been investigated in any study. Also, its reliability is determined only in neurogenic oropharyngeal dysphagia (15). The P-DHI showed good internal consistency (Cronbach's $\alpha=0.88)$ and strong test-retest reliability $($ ICC $=0.98)(15)$.

\section{Objectives}

Thus the purposes of this study were to investigate the factor structure of the P-DHI and to assess its consistency and reliability in patients with a wide variety of oropharyngeal dysphagia.

\section{Methods}

\subsection{Participants and Study Design}

This study was approved by the Ethical Committee of Tehran University of Medical Sciences (code: 93/d/260/683).
Before any examination, the informed consent form was completed by the patients to participate in this study. Patients were recruited from neurology and ear, nose, and throat clinics of Tehran University of Medical Sciences by convenient sampling. All patients with oropharyngeal dysphagia; either mechanical or neurogenic, were assessed by a speech-language pathologist using the Persian Northwestern dysphagia patient check sheet (P-NDPCS) to enter the study (18). The P-NDPCS is composed of 28 items and five medical histories, behavioral, gross motor function, oral motor evaluation, and evaluation of swallowing sections. The P-NDPCS showed good internal consistency (Cronbach's $\alpha=0.955$ ) and strong test-retest reliability $($ ICC $=0.956)(18)$. Also, patients who understood written or spoken Persian were included. We used the MiniMental State examination (MMSE) test to evaluate cognitive abilities and we excluded patients with cognitive impairments (a score of $<23$ ). The MMSE is a brief test to measure cognitive impairments, including memory, calculation, orientation, language, and attention (19). Finally, patients who met the inclusion criteria filled out P-DHI. To evaluate the test-retest reliability, the P-DHI was administered to 20 patients twice with an interval of 2 weeks.

In this study, The P-DHI was administered to 100 patients (47 men and 53 women) with oropharyngeal dysphagia. The mean age was $55.69 \pm 15.04$ years (range: 24 - 84 years), mean education level was $8.64 \pm 4.92$ years (range: 1 - 18 years), and mean disease duration was 14.07 \pm 17.45 months (range: 1 - 120 months). Of the patients, 57\% had neurological diseases (cerebrovascular diseases, Parkinson's disease, amyotrophic lateral sclerosis, multiple sclerosis, and myasthenia gravis) and $43 \%$ had head and neck disorders (head and neck cancer, vocal fold paralysis and phono surgery). The distribution of the patients according to self-reported severity of dysphagia was as follows: normal (11\%), mild (17\%), moderate (60\%), and severe (12\%).

\subsection{Persian Dysphagia Handicap Index}

The P-DHI items are scored on a 3-point Likert scale (never $=0$, sometimes $=2$, and always $=4$ ). Total score and subscales scores can range as follows: P-DHI total (25 items, 0 -100), physical (9 items, 0 -36), functional ( 9 items, 0 -36), and emotional ( 7 items, 0 -28), with lower scores indicating greater levels of QoL. At the end of the P-DHI scale there is a question about the severity of dysphagia, 1 as normal,2 and 3 as mild, 4 and 5 as moderate, and finally 6 and 7 as severe (9). 


\subsection{Data Analysis}

The factor structure of the P-DHI was investigated using Confirmatory Factor Analysis. To assess model fit, we used the chi-square statistic $\left(\chi^{2}\right)$, relative/normed chisquare $\left(\chi^{2} / \mathrm{df}\right)$, the comparative fit index (CFI), the root mean square error of approximation (RMSEA), and the standardized root means square residual(SRMR). Typically, a non-significant chi-square value represents a good model fit. However, the chi-square statistic is highly sensitive to sample size. An alternative evaluation of the $\chi^{2}$ statistic is to examine the relative/normed chi-square $\left(\chi^{2} / \mathrm{df}\right)$ for the model. A $\chi^{2} / \mathrm{df}$ ratio of 3 or less is suggestive of a good model fit (20). Values greater than 0.90 for CFI and less than .08 for RMSEA indicate reasonably good fit (21), whereas SRMR should be below 0.10 (20). Cronbach's alpha and ICC were used to examine internal consistency and test-retest reliability, respectively. A Cronbach's alpha value of $0.7-0.8,0.8-0.9$, and $>0.9$ are considered as acceptable, good, and excellent internal consistency, respectively (22). For ICC, values of $<0.5,0.5-0.75,0.75-0.9$ and $>$ 0.9 are considered as poor, moderate, good, and excellent reliability, respectively (23).

Moreover, the relationship between P-DHI and demographic/clinical characteristics was investigated using Pearson's correlation coefficient, independent samples $t$ test, and one-way ANOVA followed by the Tukey post-hoc test. All statistical analyses were carried out using SPSS version 17.0 for windows, except for the CFA, which was conducted using Lisrel 8.80. The level of significance was set at 0.05 , and all statistical tests were two-sided.

\section{Results}

\subsection{Descriptive Statistics}

The mean total P-DHI score and the subscales' scores are presented in Table 1. According to scoring instruction, lower scores indicating better QoL. The mean total score was $33.93 \pm 18.05$. The lowest and highest scores were observed for Emotional (9.40 \pm 5.75) and Physical (13.29 \pm 7.12) subscales, respectively. The correlation coefficient between total score and its subscales were high, as well as between the subscales (ranging from 0.542 to 0.909 , all $\mathrm{P}=$ 0.0001).

\subsection{Reliability Analysis}

For test-retest reliability, The P-DHI was readministered to 20 subjects of this study group one or two weeks later. The internal consistency (Cronbach's alpha) of the P-DHI were as follows: Physical subscale (9 items, 0.751), Functional subscale (9 items, 0.836), Emotional subscale (7 items, 0.773), and the total score (25 items, 0.900). The testretest reliability of the P-DHI in 20 patients using ICC was 0.984 for the total score, 0.952 for Physical 0.988 for Functional, 0.957 for the Emotional subscale (Table 2).

\subsection{Confirmatory Factor Analysis}

The CFA was undertaken to examine the fitness of the second-order three-factor model of the P-DHI. The fit indices indicated an acceptable fit of the data to the model $\left(\chi^{2}=484.61, \mathrm{df}=273, \mathrm{P}=0.0001 ; \chi^{2} / \mathrm{df}=1.77 ; \mathrm{CFI}=0.901\right.$; RMSEA $=0.088$ and SRMR $=0.010)$. As presented in Figure 1 , all standardized factor loadings were significant and in the expected direction, ranging from 0.32 to 0.79 .

\subsection{Relationship of P-DHI with Demographic Characteristics}

Table 3 shows the relationship between the demographic/clinical characteristics and P-DHI using univariate analysis. According to the correlation analysis, total P-DHI score were uncorrelated with age $(\mathrm{r}=0.123, \mathrm{P}=0.223)$, level of education $(\mathrm{r}=-0.160, \mathrm{P}=0.113)$, and duration of disease $(r=0.154, P=0.126)$. Scores also did not differ significantly by sex $(\mathrm{P}=0.936)$. Regarding the self-reported severity of dysphagia, the total P-DHI score was significantly positively correlated with the severity of dysphagia $(\mathrm{P}<0.001)$. Moreover, as presented in Table 3, the same results were obtained for Physical, Functional, and Emotional subscales.

\section{Discussion}

The purpose of this study was to examine the factor structure and validation of the P-DHI in a sample of Iranian patients with oropharyngeal dysphagia. P-DHI is a dysphagia-specific instrument and opposed to similar generic measures, it only limits the factors affecting the quality of life to dysphagia and not the other stressful events. In the present study, the mean P-DHI score was which is higher than what was reported by Silbergleit et al. (9) and Farahat et al. (14). Moreover, the highest PDHI subscales score (worst QoL) was observed for the Physical subscale. This finding is in agreement with the results reported by Silbergleit et al. (9), Farahat et al. (14), and Barzegar-Bafrooei et al. (15). It can be explained in this way that individuals with dysphagia are most familiar with their physical symptoms over the others and they tend to associate them with a swallowing disorder.

This is the first study, to the best of our knowledge, examining the factor structure of DHI by CFI. The secondorder three-factor model of P-DHI was tested. In general, this model provided an acceptable fit to data and all standardized factor loadings were statistically significant. 


\begin{tabular}{lcccc}
\hline Table 1. Means, Standard Deviations, and Correlations Between Total and Sub-Scale Scores of Persian Dysphagia Handicap Index & \\
\hline Score & Values & Total & Physical & Functional \\
\hline Total & $33.93 \pm 18.05$ & 1 & & Emotional \\
Physical & $13.29 \pm 7.12$ & $0.848^{\mathrm{b}}$ & 1 & 1 \\
Functional & $11.24 \pm 7.95$ & $0.909^{\mathrm{b}}$ & $0.637^{\mathrm{b}}$ & $0.681^{\mathrm{b}}$ \\
Emotional & $9.40 \pm 5.75$ & $0.832^{\mathrm{b}}$ & $0.542^{\mathrm{b}}$ & 1 \\
\hline
\end{tabular}

Abbreviation: SD, standard deviation.

${ }^{\mathrm{a}}$ Values are expressed as mean $\pm \mathrm{SD}$.

${ }^{\mathrm{b}} \mathrm{P}<0.001$.

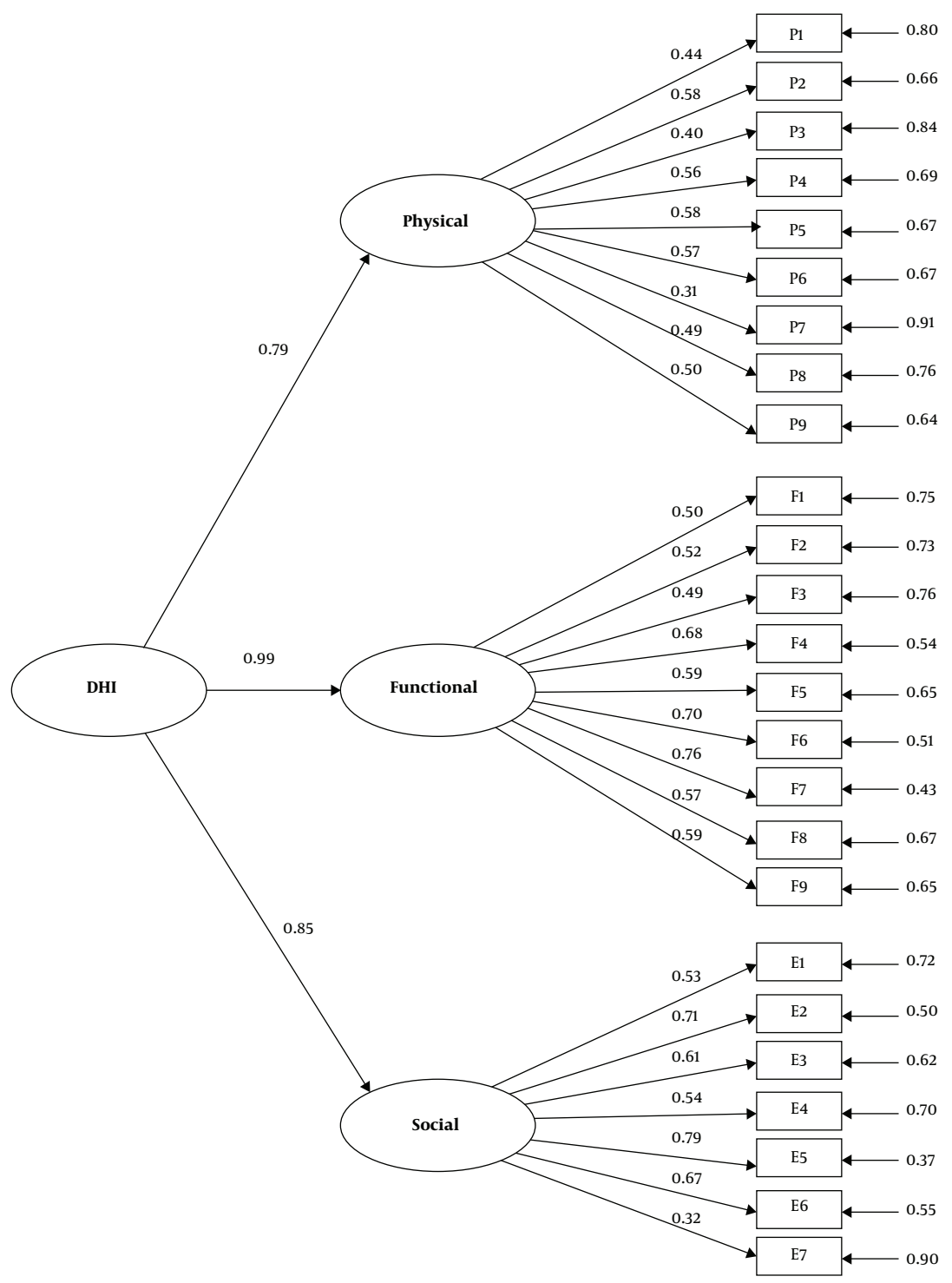


Table 2. Reliability Analysis of the Total and Subscale Scores of Persian Dysphagia Handicap Index in Patients with Oropharyngeal Dysphagia

\begin{tabular}{lccc}
\hline \multirow{2}{*}{ Score } & \multirow{2}{*}{$\begin{array}{c}\text { Number of } \\
\text { Items }\end{array}$} & \multicolumn{2}{c}{ Reliability Analysis } \\
\cline { 3 - 4 } & & Alpha a & ICC b \\
\hline Physical & 9 & 0.751 & 0.952 \\
\hline Functional & 9 & 0.836 & 0.988 \\
\hline Emotional & 7 & 0.773 & 0.957 \\
Total & 25 & 0.900 & 0.984 \\
\hline
\end{tabular}

Abbreviation: ICC, intraclass correlation coefficient.

${ }^{a}$ Cronbach's alpha sample size, $\mathrm{n}=100$.

${ }^{\mathrm{b}}$ ICC sample size, $\mathrm{n}=20$; average test-retest interval $=14$ days.

The scale and its subscales showed acceptable to excellent internal consistency. This result is in line with previous investigations that have reported acceptable to excellent internal consistency for this scale $(9,14,16)$. The scale and its subscales also showed excellent test-retest reliability, indicating that the scores of the P-DHI were stable after the two-week interval. This is in agreement with previous studies that have reported adequate test-retest reliability for DHI (9,14-16).

There was a positive correlation between the overall P-DHI scores and severity of dysphagia scores; in other words, the higher the severity, the greater the DHI score. This is also in line with previous findings from Silbergleit et al. (9), Farahat et al. (14), Barzegar-Bafrooei et al. (15), Oda et al. (16) and Shapira-Galitz et al. (17). P-DHI score was not significantly related to age, sex, level of education, and duration of disease.

\subsection{Limitations}

It is plausible that some limitations could have influenced the results obtained. First, the sample size was relatively small given the wide confidence intervals thus the findings of the study should be interpreted with caution. Second, because there were no other tests in Persian to assess the quality of life in dysphagic patients, we could not measure the relationship between the scores of P-DHI and the scores of other tests, in other words, convergent validity has not been evaluated in this study. Finally, this study design was cross-sectional, thus it is not possible to conclude the nature of relationships between P-DHI and demographics or clinical variables.

\subsection{Conclusions}

In general, it can be concluded that 25 questions of the Persian version of the swallowing disability index, which are summarized in three physical, emotional, and functional factors, are related to each other by factor analysis method and can determine the effect of dysphagia on patients' quality of life. Confirmatory factor analysis (CFA) revealed that the three-factor model of P-DHI demonstrated a superior fit to the data and the CFA findings provide additional support for the three-factor structure of the P-DHI and the use of the subscales as distinct variables. The Cronbach alpha values showed that P-DHI has good reliability. The results supported the notion that the P-DHI is a valid and reliable measurement instrument in predicting effects of dysphagia on quality of life and can be used as a tool that has good for clinical and research activities of dysphagia

\section{Acknowledgments}

We thank the patients who participated in this study, and the Tehran University of Medical Sciences, Tehran, Iran for supporting this study, grant number: 9211360002.

\section{Footnotes}

Authors' Contribution: SM did conception and design, analysis and interpretation of the data, and drafting of the article. EBB, JB, and ARK did conception and design, collection and assembly of data, interpretation of the data, drafting of the article. FF performed the medical examination of the patient and was a major contributor in writing the manuscript. AR and SK did analysis and interpretation of the data and contribute in writing the manuscript. All authors approved the final version of the article for submission

Conflict of Interests: The authors declare that they have no competing interests.

Ethical Approval: The Ethics Committee of Tehran University of Medical Sciences, Tehran, Iran, approved this study (reference number: 93/d/260/683).

Funding/Support: Tehran University Medical Sciences supported this research (grant number: 9211360002).

Informed Consent: Agreement to participate and a signed consent form were obtained from all patients before data collection 


\begin{tabular}{|c|c|c|c|c|c|c|c|c|}
\hline & \multicolumn{2}{|c|}{ Physical } & \multicolumn{2}{|c|}{ Functional } & \multicolumn{2}{|c|}{ Emotional } & \multicolumn{2}{|c|}{ DHI Total } \\
\hline & rValue & P Value & rValue & PValue & rValue & PValue & rValue & P Value \\
\hline Age & 0.123 & 0.221 & 0.104 & 0.304 & 0.089 & 0.378 & 0.123 & 0.223 \\
\hline Level of education & -0.145 & 0.149 & -0.165 & 0.102 & -0.093 & 0.355 & -0.160 & 0.113 \\
\hline $\begin{array}{l}\text { Duration of } \\
\text { disease }\end{array}$ & 0.154 & 0.125 & 0.097 & 0.339 & 0.159 & 0.114 & 0.154 & 0.126 \\
\hline Sex & & 0.837 & & 0.926 & & 0.895 & & 0.936 \\
\hline Male & $13.45 \pm 7.37$ & & $11.32 \pm 7.93$ & & $9.32 \pm 5.92$ & & $34.09 \pm 18.89$ & \\
\hline Female & $13.15 \pm 6.96$ & & $11.17 \pm 8.05$ & & $9.47 \pm 5.66$ & & $33.79 \pm 17.46$ & \\
\hline $\begin{array}{l}\text { Severity of } \\
\text { disorder }\end{array}$ & & $<0.001$ & & $<0.001$ & & $<0.001$ & & $<0.001$ \\
\hline Normal & $5.64 \pm 1.96$ & & $4.00 \pm 1.26$ & & $4.00 \pm 2.19$ & & $13.64 \pm 3.44$ & \\
\hline Mild & $9.24 \pm 3.90$ & & $5.29 \pm 3.60$ & & $5.18 \pm 3.54$ & & $19.71 \pm 6.04$ & \\
\hline Moderate & $13.57 \pm 5.67$ & & $11.70 \pm 6.09$ & & $9.70 \pm 4.05$ & & $34.97 \pm 10.95$ & \\
\hline Severe & $24.67 \pm 5.74$ & & $24.00 \pm 8.00$ & & $18.83 \pm 5.75$ & & $67.50 \pm 16.32$ & \\
\hline Type of disorders & & 0.988 & & 0.737 & & 0.481 & & 0.714 \\
\hline Neurological & $13.28 \pm 6.96$ & & $11.47 \pm 7.61$ & & $9.75 \pm 5.11$ & & $34.51 \pm 16.35$ & \\
\hline $\begin{array}{l}\text { Head and } \\
\text { neck }\end{array}$ & $13.30 \pm 7.42$ & & $10.93 \pm 8.47$ & & $8.93 \pm 6.54$ & & $33.16 \pm 20.27$ & \\
\hline
\end{tabular}

Abbreviations: SD, standard deviation; r, Pearson correlation coefficient.

${ }^{\mathrm{a}}$ Values are expressed mean $\pm \mathrm{SD}$.

\section{References}

1. Rofes L, Arreola V, Almirall J, Cabre M, Campins L, Garcia-Peris P, et al. Diagnosis and management of oropharyngeal Dysphagia and its nutritional and respiratory complications in the elderly. Gastroenterol Res Pract. 2011;10:256-65. doi: 10.1155/2011/818979. [PubMed: 20811545]. [PubMed Central: PMC2929516]

2. Chen PH, Golub JS, Hapner ER, Johns 3rd MM. Prevalence of perceived dysphagia and quality-of-life impairment in a geriatric population. Dysphagia. 2009;24(1):1-6. doi: 10.1007/s00455-008-9156-1. [PubMed: 18368451].

3. Davis LA. Quality of life issues related to dysphagia.Top Geriatr Rehabil. 2007;23(4):352-65. doi:10.1097/01.tgr.0000299163.46655.48.

4. Guyatt GH, Feeny DH, Patrick DL. Measuring health-related quality of life. Ann Intern Med. 1993;118(8):622-9. doi: 10.7326/0003-4819-118-8199304150-00009. [PubMed: 8452328].

5. Martino R, Martin RE, Black S. Dysphagia after stroke and its management. CMAJ. 2012;184(10):1127-8. doi: 10.1503/cmaj.101659. [PubMed: 22125328]. [PubMed Central: PMC3394815].

6. Swan K, Speyer R, Heijnen BJ, Wagg B, Cordier R. Living with oropharyngeal dysphagia: effects of bolus modification on health-related quality of life-a systematic review. Qual Life Res. 2015;24(10):2447-56. doi: 10.1007/s11136-015-0990-y. [PubMed: 25869989].

7. Finizia C, Rudberg I, Bergqvist H, Ryden A. A cross-sectional validation study of the Swedish version of SWAL-QOL. Dysphagia. 2012;27(3):32535. doi: 10.1007/s00455-011-9369-6. [PubMed: 22006366].

8. Keage M, Delatycki M, Corben L, Vogel A. A systematic review of selfreported swallowing assessments in progressive neurological disorders. Dysphagia. 2015;30(1):27-46. doi: 10.1007/s00455-014-9579-9. [PubMed: 25280814].

9. Silbergleit AK, Schultz L, Jacobson BH, Beardsley T, Johnson AF. The Dysphagia handicap index: development and validation. Dysphagia. 2012;27(1):46-52. doi: 10.1007/s00455-011-9336-2. [PubMed: 21424584].
10. Chen AY, Frankowski R, Bishop-Leone J, Hebert T, Leyk S, Lewin J, et al. The development and validation of a dysphagia-specific qualityof-life questionnaire for patients with head and neck cancer: the MD Anderson dysphagia inventory. Arch Otolaryngol. 2001;127(1):870-6.

11. McHorney CA, Bricker DE, Kramer AE, Rosenbek JC, Robbins J, Chignell $\mathrm{KA}$, et al. The SWAL-QOL outcomes tool for oropharyngeal dysphagia in adults: I. Conceptual foundation and item development. Dysphagia. 2000;15(3):115-21. doi: 10.1007/s004550010012. [PubMed: 10839823].

12. Belafsky PC, Mouadeb DA, Rees CJ, Pryor JC, Postma GN, Allen J, et al Validity and reliability of the eating assessment tool (eat-10). Ann Otol Rhinol Laryngol. 2008;117(12):919-24. doi: 10.1177/000348940811701210. [PubMed: 19140539].

13. Rhinol RLO. The "Deglutition Handicap Index" a self-adminitrated dysphagia-specific quality of life questionnaire: sensibility to change. Rev Laryngol Otol Rhinol. 2011;132(1):3-7.

14. Farahat M, Malki KH, Mesallam TA, Bukhari M, Alharethy S. Development of the arabic version of dysphagia handicap index (dhi). Dys phagia. 2014;29(4):459-67. doi: 10.1007/s00455-014-9528-7. [PubMed: 24842336].

15. Barzegar-Bafrooei E, Bakhtiary J, Khatoonabadi AR, Fatehi F, Maroufizadeh S, Fathali M. Validation of the Persian version of the dysphagia handicap index in patients with neurological disorders. Curr J Neurol. 2016;15(3):128-32.

16. Oda C, Yamamoto T, Fukumoto Y, Nakayama K, Sato M, Murata M, et al. Validation of the japanese translation of the dysphagia handicap index. Patient Prefer Adherence. 2017;11:193-8. doi: 10.2147/PPA.S126052. [PubMed: 28203063]. [PubMed Central: PMC5295790].

17. Shapira-Galitz Y, Drendel M, Yousovich-Ulriech R, ShtreifflerMoskovich L, Wolf M, Lahav Y. Translation and validation of the dysphagia handicap index in hebrew-speaking patients. Dysphagia 2019;34(1):63-72. doi: 10.1007/s00455-018-9914-7. [PubMed: 29882103]. 
18. Bakhtiyari J, Salmani M, Noruzi R, Sarraf P, Barzegar E, Mirmohammadkhani M. Translation, cross-cultural adaptation, validation and reliability of the northwestern dysphagia patient check sheet (NDPCS) in Iran. Iran J Otorhinolaryngol. 2018;30(97):97.

19. Ansari NN, Naghdi S, Hasson S, Valizadeh L, Jalaie S. Validation of a Mini-Mental State Examination (MMSE) for the Persian population: a pilot study. Appl Neuropsychol. 2010;17(3):190-5. doi: 10.1080/09084282.2010.499773. [PubMed: 20799110].

20. Kline RB. Principles and practice of structural equation modeling. Guilford publications; 2015.
21. Hu L, Bentler PM. Cutoff criteria for fit indexes in covariance structure analysis: Conventional criteria versus new alternatives. Struct Equ Modeling. 1999;6(1):1-55. doi: 10.1080/10705519909540118.

22. Nunnally JC. Psychometric theory. 3nd ed. New York: McGraw-hill education; 1994.

23. Koo TK, Li MY. A guideline of selecting and reporting intraclass correlation coefficients for reliability research. J Chiropr Med. 2016;15(2):155-63. doi: 10.1016/j.jcm.2016.02.012. [PubMed: 27330520]. [PubMed Central: PMC4913118]. 